\title{
Effect of Induced Nodule Senescence on Parameters Related to Dinitrogen Fixation, Bacteroid Size and Nucleic Acid Content
}

\author{
By ALAN S. PAAU* AND JOE R. COWLES \\ Department of Biology, University of Houston, Houston, Texas 77004, U.S.A.
}

(Received 6 June 1978; revised 31 August 1978)

\begin{abstract}
Darkness and treatment with combined nitrogen $\left(\mathrm{NH}_{4} \mathrm{Cl}\right.$ or $\left.\mathrm{KNO}_{3}\right)$ were used to induce nodule senescence in alfalfa and soybeans. Nodule senescence was assessed by determinations of the acetylene-reducing activity and leghaemoglobin and sugar contents of the nodules. Bacteroids from nodules of the treated plants were compared using flow microfluorimetry. Upon induced nodule senescence, alfalfa bacteroids decreased both in nucleic acid content and cell size while the soybean bacteroids were essentially unaffected.
\end{abstract}

\section{INTRODUCTION}

Dinitrogen fixation by Rhizobium-legume associations involves a complex physiological interplay between the symbiotic rhizobia (bacteroids) and the host plants. The reduction of dinitrogen occurs in the bacteroids while the required energy and reductants are supplied by the plant through photosynthesis. Ching et al. (1975) demonstrated in soybean nodules that if photosynthesis became limiting, acetylene reduction, ATP and sucrose contents and the energy charge of the nodules sharply decreased. In a separate study, Chen \& Phillips (1977) have shown that combined nitrogen $\left(\mathrm{NH}_{4} \mathrm{Cl}\right.$ or $\left.\mathrm{KNO}_{3}\right)$ also caused a sharp decrease in acetylene reduction in pea nodules. The reason for this effect is not known although various explanations have been suggested (Oghoghorie \& Pate, 1971; Rigaud, 1976; Rigaud \& Puppo, 1977).

Previous research from this laboratory (Paau et al., 1978) has shown that the basal portion of the 6 week or older alfalfa nodule has very low acetylene reduction capability in comparison with the middle nodule region and that bacteroids in this basal region have considerably less nucleic acid than bacteroids of similar size in the middle nodule regions. The bacteroids in the basal region apparently have begun senescence. While the effects of limited light and combined nitrogen have been shown to reduce parameters related to dinitrogen fixation in the nodules, their specific effect on bacteroid senescence has not been reported. In this communication, we demonstrate the effect of darkness and treatment with combined nitrogen on the nucleic acid content of bacteroids from alfalfa and soybean nodules. Changes in the acetylene-reducing activity of the nodules and in the leghaemoglobin and sugar contents of the nodule cytosol are also reported.

\section{METHODS}

The maintenance and growth conditions of free-living Rhizobium meliloti F28 and Rhizobium japonicum 705 were as previously described (Cowles et al., 1969). Alfalfa (Medicago sativa Buffalo) and soybean (Glycine max Chippewa) plants were inoculated 1 week after germination with cultures of $R$. meliloti F28 and $R$. japonicum 705, respectively. The soybean and alfalfa nodules were harvested 8 and 6 weeks after inoculation,

* Present address: Department of Bacteriology, University of Wisconsin, Madison, Wisconsin 53706, U.S.A. 
respectively. The times at which treatments were initiated were chosen so that the control and treated plants were harvested on the same day. For dark treatments, the plants were covered with black plastic bags (NAPCO, $1 \times 0.9 \mathrm{~m}$ ) on the appropriate day before harvest. The control plants were covered with transparent plastic bags. The black bags transmitted 4,6 and 7.5\% of incident light at 450, 550 and $680 \mathrm{~nm}$, respectively; the transparent bags transmitted 62,60 and $72 \%$ of incident light at these wavelengths. For combined nitrogen treatments, $500 \mathrm{ml}$ of $100 \mathrm{mM}-\mathrm{NH}_{4} \mathrm{Cl}$ or $\mathrm{KNO}_{3}$ was applied to the plants on the appropriate initial day and continued every other day until harvest.

The harvested nodules were first assayed for acetylene-reducing activity by gas chromatography (Hardy et al., 1968). The gas phase consisted of $65 \%$ helium, $24 \%$ oxygen and $10 \%$ acetylene. Afterwards, the nodules were homogenized and the bacteroids were harvested by differential centrifugation (Cowles \& Evans, 1968; Cowles et al., 1969). The supernatant (nodule cytosol) was used for determinations of leghaemoglobin, glucose and sucrose contents. Leghaemoglobin was determined spectrophotometrically, using human haemoglobin as a standard, by an assay modified from that of Johnson \& Hume (1973): $2.5 \mathrm{ml}$ of nodule cytosol was combined with $0.5 \mathrm{ml}$ of $5 \mathrm{M}-\mathrm{KOH}$ containing $0.1 \mathrm{~g}$ sodium dithionite and $A_{555}$ was determined. Glucose was determined using the 'glucose-stat' procedure as outlined by Worthington Biochemical Co. Sucrose was hydrolysed by invertase and the glucose formed was quantified by the 'glucose-stat' procedure. The nucleic acid content and cell size of the individual bacteroids were determined from ethidium bromide fluorescence and light-scattering properties, respectively, in a flow microfluorimeter (Paau et al., 1977a,b). The bacteroids were fixed overnight in $65 \%(\mathrm{v} / \mathrm{v})$ ethanol and stained with $0.005 \%(\mathrm{w} / \mathrm{v})$ ethidium bromide in $1.12 \%(\mathrm{w} / \mathrm{v})$ sodium citrate (Paau et al., 1977a). For DNA analysis, the fixed cells were digested with RNAase before staining (Paau et al., 1977a). The reported results are averages of duplicate experiments.

\section{RESULTS}

\section{Acetylene-reducing activity and leghaemoglobin, sucrose and glucose contents in nodules}

The acetylene-reducing activity in alfalfa nodules had decreased by more than $50 \%$ after $1 \mathrm{~d}$ in darkness and by more than $80 \%$ after $3 \mathrm{~d}$ (Fig. $1 \mathrm{a}$ ). About $70 \%$ of the initial activity was retained in soybean nodules after $4 \mathrm{~d}$ in darkness (Fig. $1 d$ ). Acetylene-reducing activity was not detectable in nodules of either plant after $6 \mathrm{~d}$. The decrease in acetylenereducing activity in soybean nodules in the dark is consistent with that described by Ching et al. (1975). Treatment with $\mathrm{KNO}_{3}$ caused a rapid and similar decrease in acetylenereducing activity in both alfalfa and soybean nodules; more than $50 \%$ of the initial activity was lost after $1 \mathrm{~d}$ (Fig. $1 b, e$ ). A similar response was caused by the $\mathrm{NH}_{4} \mathrm{Cl}$ treatments except the rate of loss was somewhat slower than with $\mathrm{KNO}_{3}$ (Fig. $1 c, f$ ).

The leghaemoglobin contents of alfalfa and soybean nodules also decreased in response to the dark and combined nitrogen treatments. After $11 \mathrm{~d}$ of treatment, the leghaemoglobin content remained above $50 \%$ of that of the control nodules for both the alfalfa and soybean systems. The decrease in leghaemoglobin content during senescence probably influenced the rate of acetylene reduction (Swaraj \& Garg, 1977).

The changes in sucrose and glucose contents caused by darkness or treatment with combined nitrogen are shown in Fig. 2. In both alfalfa and soybean nodules, the sucrose content had decreased by more than $30 \%$ after $1 \mathrm{~d}$ in darkness and was less than $25 \%$ of the content in control plants after $11 \mathrm{~d}$ (Fig. $2 a, c$ ). The decrease in glucose content in the dark was initially slower than that of sucrose but it then became more rapid. Treatment of alfalfa plants with $\mathrm{KNO}_{3}$ caused the sucrose content to decrease initially (about $40 \%$ in $8 \mathrm{~d}$ ) and then stabilize (Fig. 2b). Sucrose contents in soybean nodules did not change appreciably (Fig. $2 d$ ). The glucose content in alfalfa nodules had increased twofold by $1 \mathrm{~d}$ after $\mathrm{KNO}_{3}$ treatment and then decreased to below the control level by $11 \mathrm{~d}$ (Fig. $2 b$ ). In soybean nodules the increase in glucose content by $1 \mathrm{~d}$ after $\mathrm{KNO}_{3}$ treatment was about fourfold. This concentration was maintained for up to $4 \mathrm{~d}$, but by $7 \mathrm{~d}$ the glucose concentration was below that of the control (Fig. $2 d$ ). Results from plants treated with $\mathrm{NH}_{4} \mathrm{Cl}$ were similar to those treated with $\mathrm{KNO}_{3}$ (data not shown). 


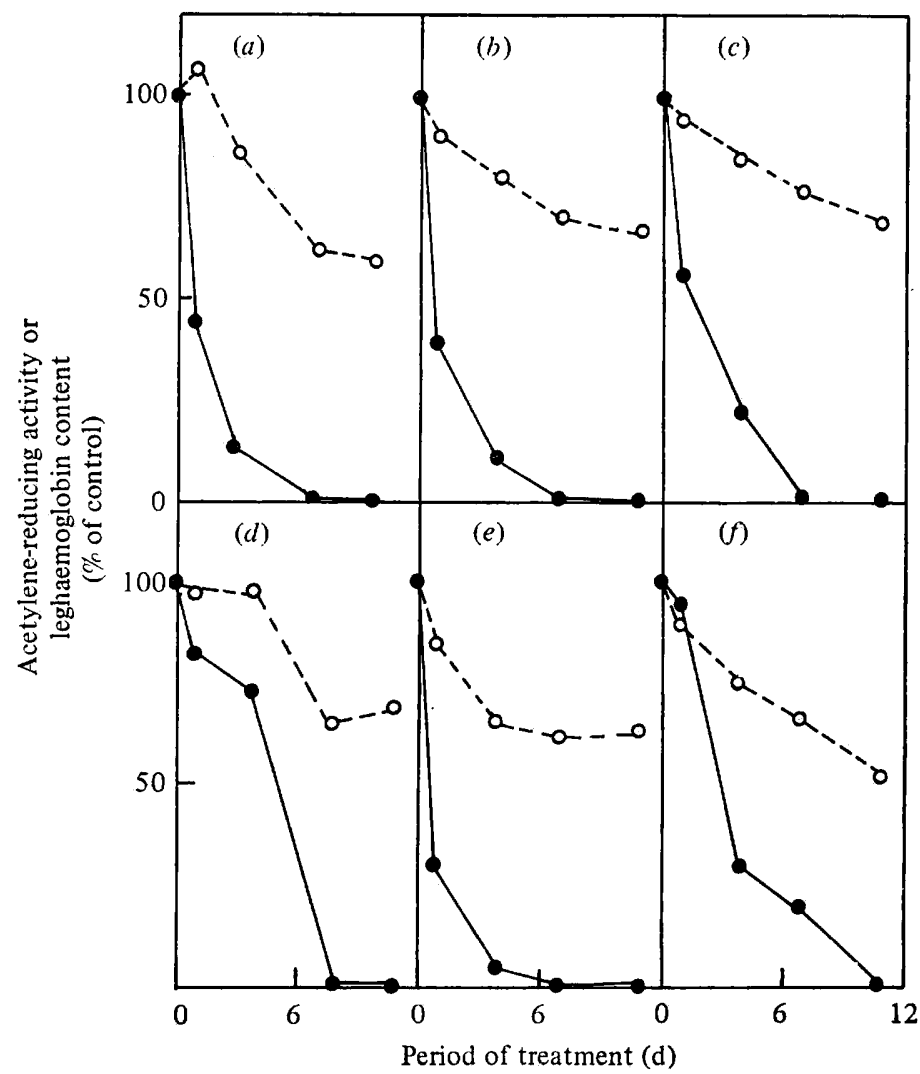

Fig. 1. Effect of darkness $(a, d), \mathrm{KNO}_{3}(b, e)$ and $\mathrm{NH}_{4} \mathrm{Cl}(c, f)$ treatments on the acetylene-reducing activity $(O)$ and leghaemoglobin content $(O)$ of alfalfa $(a, b, c)$ and soybean $(d, e, f)$ nodules. Values for the control experiments were: alfalfa, $3.16 \mathrm{mg}$ leghaemoglobin (g nodule) ${ }^{-1}$ and $4.5 \mu \mathrm{mol} \mathrm{C}_{2} \mathrm{H}_{2}$ reduced $\mathrm{g}^{-1} \mathrm{~h}^{-1}$; soybean, $2 \mathrm{mg}$ leghaemoglobin (g nodule) ${ }^{-1}$ and $2 \cdot 6 \mu \mathrm{mol} \mathrm{C}_{2} \mathrm{H}_{2}$ reduced $\mathrm{g}^{-1} \mathrm{~h}^{-1}$.

\section{Nucleic acid content of alfalfa bacteroids}

The effect of darkness or treatment with combined nitrogen on the nucleic acid content of alfalfa nodule bacteroids was determined by flow microfluorimetry of isolated bacteroids. These analyses revealed a slight decrease in the average DNA content in bacteroids with increasing periods of darkness (Fig. $3 b$ to $f$ ). The number of bacteroids with a high DNA content (100 units or higher) decreased in proportion to the period spent in darkness. The alfalfa bacteroid DNA content remained as high as that of the free-living rhizobia even after $10 \mathrm{~d}$ in the dark (Fig. $3 a, f$ ). If bacteroids from the control plants were not treated with RNAase before staining, the intensity of the fluorescence was generally greater in the untreated bacteroids (compare Fig. $3 b, h$ ). This difference is due presumably to the tertiary structures of the bacteroid RNA resulting in the binding of ethidium bromide. After $3 \mathrm{~d}$ in the dark, the RNA component of the ethidium bromide fluorescence from bacteroids had completely disappeared (Fig. $3 h$ to $l$ ). This indicates that the dark treatment rapidly decreases the RNA content or changes its structure. There was no significant change in ethidium bromide fluorescence by RNA digestion in the free-living rhizobia (Fig. $3 a, g$ ).

Treatment of alfalfa plants with $\mathrm{KNO}_{3}$ or $\mathrm{NH}_{4} \mathrm{Cl}$ led to a decrease in the DNA and RNA contents of bacteroids similar to that caused by darkness (data not shown). The principal difference between the combined nitrogen and dark treatments was that the decrease in the RNA component of the ethidium bromide fluorescence was comparatively slower in the 


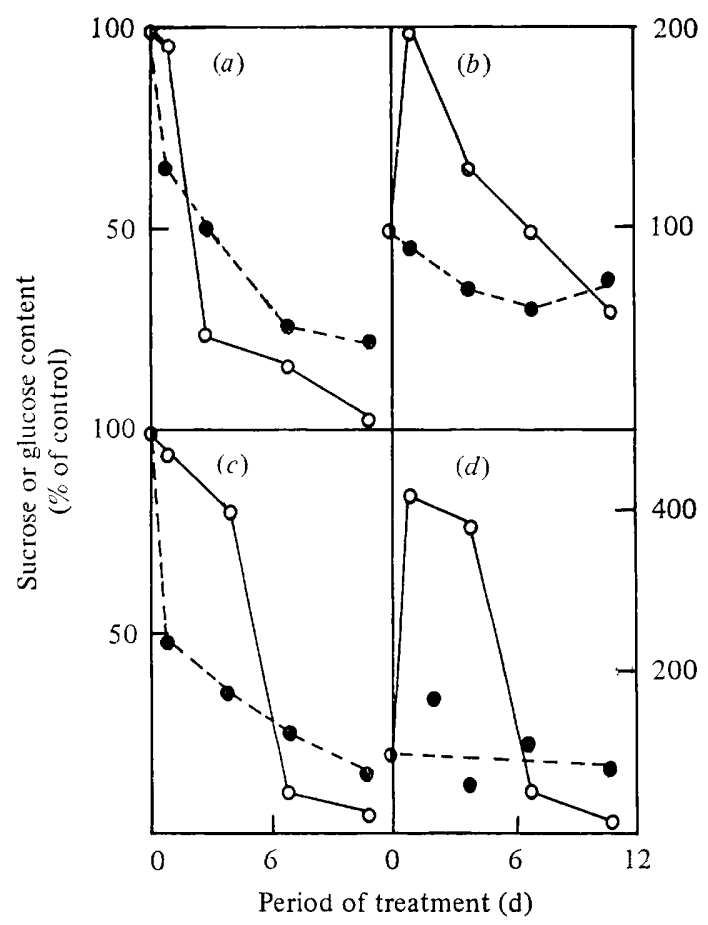

Fig. 2. Effect of darkness $(a, c)$ and $\mathrm{KNO}_{3}(b, d)$ treatments on the sucrose $(\bigcirc)$ and glucose $(\bigcirc)$ contents of alfalfa $(a, b)$ and soybean $(c, d)$ nodules. (Left-hand scales refer to $a$ and $c$, right-hand scales to $b$ and $d$.) Values for the control experiments were: alfalfa, $3 \mathrm{mg}$ sucrose and $0 \cdot 1 \mathrm{mg}$ glucose (g nodule $)^{-1}$; soybean, $5 \mathrm{mg}$ sucrose and $0.2 \mathrm{mg}$ glucose (g nodule $)^{-1}$.

nitrogen-treated plants; the decrease in the RNA component was not complete until about $7 \mathrm{~d}$ after the initiation of treatments.

\section{Nucleic acid content of soybean bacteroids}

Bacteroids isolated from soybean nodules have a similar DNA content to free-living $R$.japonicum. Soybean plants subjected to darkness or treated with combined nitrogen for $11 \mathrm{~d}$ did not show any significant change $(<4 \%)$ in the bacteroid DNA content. The results of experiments involving $\mathrm{NH}_{4} \mathrm{Cl}$ treatments are shown in Fig. 4. The RNA contribution to the ethidium bromide fluorescence in the soybean bacteroids was less than $2 \%$ and did not vary significantly with either treatment.

\section{Bacteroid size in alfalfa and soybean bacteroids}

The bacteroid populations isolated from soybean and alfalfa nodules were analysed by flow microfluorimetry using the light-scattering signals (Paau et al., 1977a). Soybean bacteroids were similar in size to free-living $R$. japonicum, and treatment of soybean plants with either darkness (Fig. $5 b, c$ ) or combined nitrogen (results not shown) did not significantly alter the bacteroid size. On the other hand, the majority of the bacteroids in alfalfa nodules are larger than the free-living rhizobia. Darkness (Fig. $5 e, f$ ) or treatment with combined nitrogen (results not shown) greatly decreased the number of enlarged bacteroids. The decrease in alfalfa bacteroid size, however, was not apparent until after $3 \mathrm{~d}$ of treatment. The disappearance of large bacteroids in the alfalfa nodules occurred after the substantial decrease in acetylene-reducing activity and bacteroid nucleic acid content. 


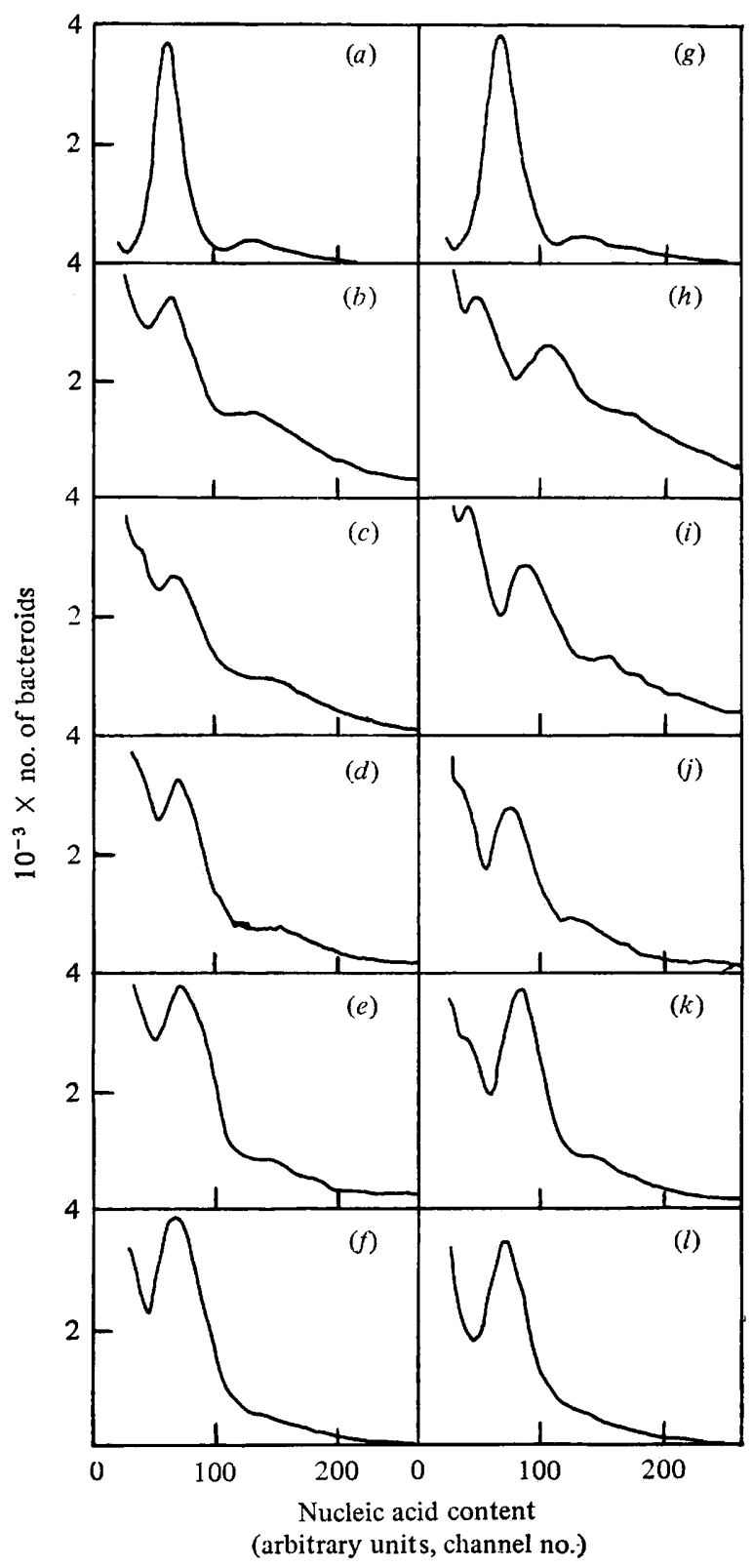

Fig. 3. Effect of darkness on the distribution of alfalfa bacteroids based on nucleic acid content: ( $a$ to $f^{\prime}$ ) distribution according to DNA content; $(g$ to $l$ ) distribution according to ethidium bromide fluorescence without RNAase digestion (see text). $(a, g)$ Free-living $R$. meliloti, at early stationary phase; $(b, h)$ untreated control; $(c, i) 1 \mathrm{~d}$ in the dark; $(d, j) 3 \mathrm{~d}$ in the dark; $(e, k) 7 \mathrm{~d}$ in the dark; $(f, l) 10 \mathrm{~d}$ in the dark. The channel number is directly proportional to the intensity of ethidium bromide fluorescence which is positively correlated to the DNA and total nucleic acid content of bacteroids. 


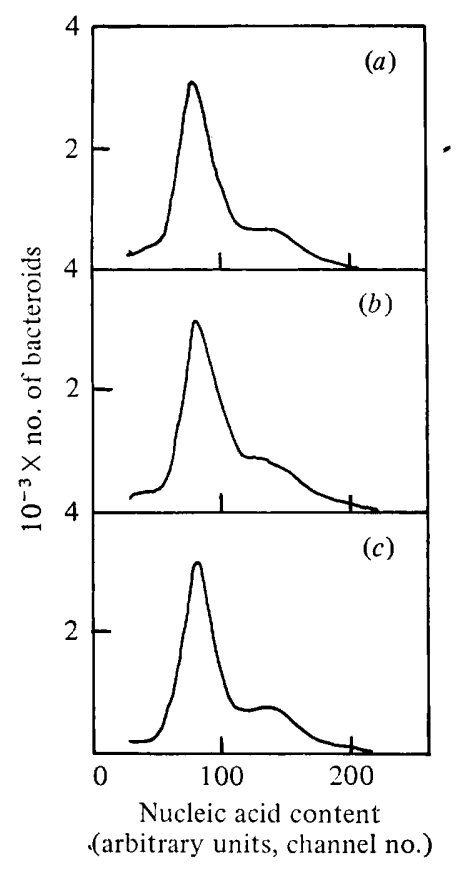

Fig. 4

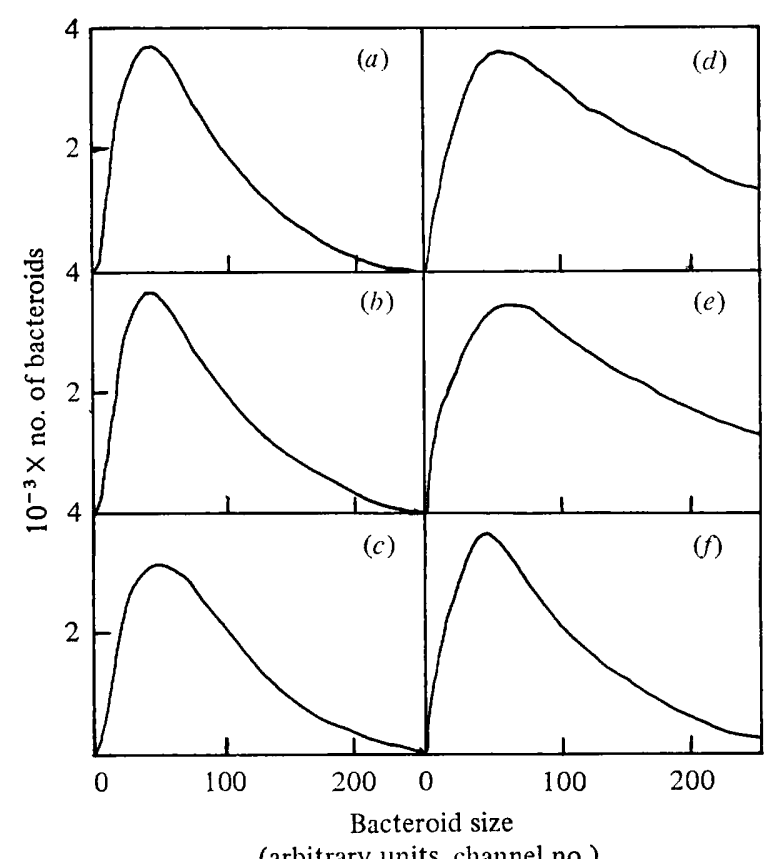

(arbitrary units, channel no.)

Fig. 5

Fig. 4. Effect of $\mathrm{NH}_{4} \mathrm{Cl}$ treatment on the distribution of soybean bacteroids based on total nucleic acid content: $(a)$ free-living $R$. japonicum, at early stationary phase; $(b)$ bacteroids from untreated plants; $(c)$ bacteroids from plant treated for $11 \mathrm{~d}$. The samples were not digested with RNAase.

Fig. 5. Effect of darkness on the distributions of soybean $(a, b, c)$ and alfalfa $(d, e, f)$ bacteroids based on cell size: $(a, d)$ untreated control; $(b, e) 3 \mathrm{~d}$ in the dark; $(c) 14 \mathrm{~d}$ in the dark; $(f) 10 \mathrm{~d}$ in the dark. The channel number is directly proportional to the intensity of the light-scattering signal which is positively correlated to the cell size.

\section{DISCUSSION}

The responses of soybean and alfalfa nodules to darkness and treatment with combined nitrogen are similar in many respects but there are significant differences. Both treatments caused a rapid (detectable in $1 \mathrm{~d}$ ) loss in nucleic acid content in the bacteroids of alfalfa plants and a subsequent reduction in bacteroid size. These changes were not seen in bacteroids of soybean nodules. Thus our observations support earlier suggestions that the two Rhizobium species (one species being involved with each plant species) are not closely related (Dixon, 1969; Gibbins \& Gregory, 1972).

The loss of acetylene-reducing activity and leghaemoglobin content in the dark and the decrease in sucrose and glucose contents was initially more rapid in the alfalfa nodules than in the soybean nodules. The rapid loss of acetylene-reducing activity in alfalfa nodules in the dark may be related to the amount of energy reserve in alfalfa nodules as well as the metabolic activity of the infecting organism. The concentration of glucose and sucrose in alfalfa nodules was only about $50 \%$ of that in soybean nodules. In addition, cultures of free-living $R$. meliloti grow three to four times as fast as cultures of $R$. japonicum. If similar differences in metabolic activity occur in the symbiotic micro-organisms, then the already small energy reserve in alfalfa nodules would be depleted faster and result in a rapid loss of acetylenereducing activity in alfalfa nodules. Interestingly, the most rapid decrease in acetylenereducing activity in the dark-treated soybean nodules begins at about the same time as the glucose content rapidly decreases. In the alfalfa nodules the glucose content begins to decline rapidly after $1 \mathrm{~d}$ in the dark. 
Although the decrease in acetylene-reducing activity in the dark has been attributed to the limited availability of energy and reductants from photosynthesis (Ching et al., 1975), the decrease caused by treatment with combined nitrogen has drawn varied explanations (Oghoghorie \& Pate, 1971; Rigaud, 1976; Chen \& Phillips, 1977; Rigaud \& Puppo, 1977). Bisseling et al. (1978) concluded that decreased nitrogenase activity in nodules of peas treated with $\mathrm{NH}_{4} \mathrm{NO}_{3}$ resulted from a decreased leghaemoglobin content. The concomitant decrease in the number of large bacteroids with a high nucleic acid content in the alfalfa nodule system with the loss in acetylene-reducing activity is also perplexing. It seems unlikely that the decrease in numbers of large bacteroids is due solely to photosynthetic limitations since the nodules of plants treated with $\mathrm{NH}_{4} \mathrm{Cl}$ and $\mathrm{KNO}_{3}$ had similar sucrose and glucose contents to the control plants up to $6 \mathrm{~d}$ after initiation of treatment. While the mechanism which regulates the degradation of large alfalfa bacteroids is unknown, it is nteresting that bacteroids responded similarly in size and nucleic acid content to darkness and to treatment with combined nitrogen.

We thank James Oro and Don Schomer for their assistance and Drs Allen Bartel and John Hungerford for making the flow microfluorimeter available.

\section{REFERENCES}

Bisseling, T., Van den Bos, R. C. \& Van Kammen, A. (1978). The effect of ammonium nitrate on the synthesis of nitrogenase and the concentration of leghemoglobin in pea root nodules induced by Rhizobium leguminosarum. Biochimica et biophysica acta 539, 1-11.

Chen, P. C. \& Phillips, D. A. (1977). Induction of root nodule senescence by combined nitrogen in Pisum sativum L. Plant Physiology 59, 440-442.

Ching, T. M., Hedtke, S., Russell, S. A. \& Evans, H. J. (1975). Energy state and dinitrogen fixation in soybean nodules of dark-grown plants. Plant Physiology 55, 796-798.

Cowles, J. R. \& Evans, H. J. (1968). Some properties of the ribonucleotide reductase from Rhizobium meliloti. Archives of Biochemistry and Biophysics 127, 770-778.

Cowles, J. R., Evans, H. J. \& Russell, S. A. (1969). $\mathrm{B}_{12}$ coenzyme-dependent ribonucleotide reductase in Rhizobium species and the effects of cobalt deficiency on the activity of the enzyme. Journal of Bacteriology 97, 1460-1465.

Dixon, R. O. D. (1969). Rhizobia (with particular reference to relationships with host plants). Annual Review of Microbiology 23, 137-158.

Gibbins, A. M. \& Gregory, K. F. (1972). Relatedness among Rhizobium and Agrobacterium species determined by the methods of nucleic acid hybridization. Journal of Bacteriology 111, 129141.

Hardy, R. W. F., Holsten, R. D., Jackson, E. K. \& Burns, R. C. (1968). The acetylene-ethylene assay for $\mathrm{N}_{2}$ fixation; laboratory and field evaluation. Plant Physiology 43, 1185-1207.

JoHnson, H. S. \& HumE, D. J. (1973). Comparison of nitrogen fixation estimates in soybeans by nodule weight, leghemoglobin content and acetylene reduction. Canadian Journal of Microbiology 19, 1165-1168.

Oghoghorie, C. G. O. \& Pate, J. S. (1971). The substrate stress syndrome of the nodulated field pea (Pisum sativum L.). In Biological Nitrogen Fixation in Natural and Agricultural Habitats, Plant and Soil Special Volume, pp. 185-202. Edited by T. A. Lie \& E. G. Mulder. The Hague: Martinus Nighoff.

PaAu, A. S., Cowles, J. R. \& Oro, J. (1977a). Flow-microfluorometric analysis of Escherichia coli, Rhizobium meliloti and Rhizobium japonicum at different stages of the growth cycle. Canadian Journal of Microbiology 23, 1165-1169.

PaAu, A. S., Lee, D. \& Cowles, J. R. (1977b). Comparison of nucleic acid content in populations of free-living and symbiotic Rhizobium meliloti by flow-microfluorometry. Journal of Bacteriology 129, 1156-1158.

PaAu, A. S., Cowles, J. R. \& Raveed, D. (1978). The development of bacteroids in alfalfa (Medicago sativa) nodules. Plant Physiology 62, 526530.

Rigaud, J. (1976). Effet de nitrates sur la fixation d'azote par les nodules de Haricot (Phaseolus vulgaris L.). Physiologie Végétale 14, 297-308.

Rigaud, J. \& PuPPo, A. (1977). Effect of nitrate upon leghemoglobin and interaction with nitrogen fixation. Biochimica et biophysica acta 497, 702706.

SwaraJ, K. \& GARG, O. P. (1977). The effect of ageing on the leghemoglobin of cowpea nodules. Physiologia plantarum 39, 185-189. 